\title{
Evaluation of errors during different phases of coronavirus disease 2019 TrueNat testing in a tertiary care hospital
}

\author{
Nisha Goyal', Abha Sharma ${ }^{2}$, Poonam Loomba ${ }^{3}$, B Mishra ${ }^{4}$, Archana Thakur \\ From ${ }^{1}$ Assistant Professor, ${ }^{2}$ Associate Professor, ${ }^{3}$ Director Professor \& Head, ${ }^{4}$ Consultant, ${ }^{5}$ Professor of Excellence, Department of Microbiology, \\ Govind Ballabh Pant Institute of Postgraduate Medical Education and Research, New Delhi, India
}

Correspondence to: Dr. Nisha Goyal, Department of Microbiology, Govind Ballabh Pant Institute of Postgraduate Medical Education and Research, New Delhi, India. E-mail: drnishagoyalucms@gmail.com

Received - 11 November $2020 \quad$ Initial Review - 15 November 2020

Accepted - 18 November 2020

\section{ABSTRACT}

Background: Although diagnostic errors have always significantly attributed to clinical and economical losses, now in ongoing coronavirus disease 2019 (COVID-19) pandemic, the impact of these errors has unquestionably increased multifold. In the present scenario, reporting of false-negative or false-positive test results may adversely affect the public health measures and government restrictive policies, established for the containment of COVID-19 in addition to enhancing the individual suffering and economic burden. Only way to reduce errors in various phases of COVID-19 testing is to evaluate them thoroughly and subsequently follow them up with effective corrective actions. Materials and Methods: The present study was conducted at a tertiary care center, approved by Indian Council of Medical Research for COVID-19 TrueNat testing. Information regarding errors and subsequent corrective actions in preanalytical, analytical, and post-analytical phases of TrueNat testing for COVID-19 were collected. Results: A total of 112 testing errors were observed in the TrueNat testing of 1108 samples during study duration. Around $58.93 \%$ errors occurred in preanalytical phase of TrueNat testing, followed by $39.29 \%$ and $1.79 \%$ errors in analytical phase and post-analytical phases, respectively. Poor quality samples constituted the predominant cause of error in preanalytical phase. Conclusion: Delivery of accurate results is of paramount importance in this COVID-19 pandemic. Thorough analysis of the various phases of COVID-19 testing will help identify and understand the errors and implement effective corrective actions.

Key words: Analytical, Coronavirus disease 2019, Preanalytical, Testing error, TrueNat

$\mathrm{T}$ The raging pandemic of novel coronavirus disease 2019 (COVID-19) has almost stopped the wheel of world. Severe acute respiratory syndrome coronavirus 2 (SARS-CoV-2) has spread its reach even to the remotest places of earth. India reported its first case of COVID-19 on January 30, 2020. Since then, India has reported a sharp rise in COVID-19 cases. As on September 17, 2020, India recorded 1,009,976 active COVID19 cases with 83,198 associated deaths [1]. India, the second most populous country in the world, is home to over 1.3 billion people. The thick population density and disparity between the distribution of health-care infrastructure in the urban and rural areas posed major hurdles in the way of India's fight against COVID-19. The World Health Organization has emphasized on the role of India in determining the worldwide trajectory of COVID-19 [2].

India implemented an early nationwide lockdown and aggressively utilized this time in enhancing the COVID-19 managing capacity by strengthening the health-care infrastructure in the country. The COVID-19 testing capacity was expanded by increasing the number of authorized COVID-19 testing government laboratories along with authorizing the capable private labs for SARS-CoV-2 testing. From National Institute of Virology, Pune, being the only lab in India authorized for SARS-CoV-2 testing in January 2020, the total operational laboratories have been scaled up to 1751 (1059 government labs and 692 private labs) in the mid of September 2020. Cartridgebased Nucleic Acid Amplification Test (CBNAAT) and TrueNat tests are also advocated by Indian Council of Medical Research (ICMR) besides conventional real-time reverse transcriptase polymerase chain reaction (rRT-PCR) for COVID-19 testing. As of the time of this writing, 891 labs are performing rRT-PCR; 737 labs TrueNat tests and 123 CBNAAT tests for COVID-19 diagnosis [3]. Till date, $60,565,728$ cumulative total samples for COVID-19 have been tested in India and 1,136,613 samples for COVID-19 have been tested on a single most recent day [4].

On April 14, 2020, ICMR issued guidance for utilization of TrueNat ${ }^{\mathrm{TM}}$ beta $\mathrm{CoV}$ as a screening test for COVID-19 [5]. TrueNat machines were originally designed for the efficient detection of Mycobacterium tuberculosis at the primary healthcare level, as these work on batteries contrary to the GeneXpert ${ }^{\mathbb{B}}$ machines which need air conditioner for proper functioning [6]. The sample for TrueNat testing is collected using virus lysis buffer that further helps in reducing the biosafety concerns $[5,6]$. The widespread familiarity with TrueNat system 
even at the peripheral centers of our country, comparatively minimal biosafety hazards and a turnaround time of $60 \mathrm{~min}$ are its major advantages over conventional rRT-PCR that needs specialized laboratory setup with skilled manpower, also involves biosecurity concerns, and has an average turnaround time of 4-5 h. However, only 1-4 samples can be tested in one run of TrueNat system and conventional rRT-PCR can test up to 90 samples in a single run. ICMR revised its guidelines for TrueNat testing for COVID-19 on May 19, 2020, and now recognized TrueNat system as a comprehensive assay capable of providing a reliable confirmation of COVID-19 in addition to being a useful screening test for the same [7].

Diagnostic errors in various phases of testing are an inherent part of any diagnostic modality and to reduce these errors to the possible minimum, their early recognition and brisk corrective action is of utmost importance. Nucleic acid amplification tests are widely trusted for the reliable diagnosis of COVID-19. Therefore, delivering the accurate test results by checking the vulnerabilities of the whole testing protocol is crucial for any COVID-19 testing facility. Diagnostic errors can occur at any step of TrueNat testing procedure. The potential errors in the preanalytical phase of testing are mismatched identification, inadequate techniques for swab collection, collection of insufficient material, use of unsuitable transport medium, and transportation of samples in containers with improperly secured caps [8]. Errors in analytical phase of TrueNat testing that may adversely affect the diagnostic accuracy include, failure of adherence to standard operating procedure (SOP), instrument malfunctioning such as cartridge or chip errors. Some issues in post-analytical phase may also jeopardize the delivery of reliable results such as erroneous reporting/entry of test results and excessive turnaround time $[9,10]$.

Although diagnostic errors have always significantly attributed to clinical and economical losses, now in ongoing COVID-19 pandemic the impact of these errors has unquestionably increased multifold [11]. In present scenario, additional to enhancing the individual suffering and economic burden, reporting of falsenegative or false-positive test results may adversely affect the public health measures and government restrictive policies established for the containment of COVID-19. A false-negative SARS-CoV-2 test report may threaten the spread of COVID-19 in the community by the unrestricted movement of an unaware COVID-19 positive case. On the contrary, false-positive SARS-CoV-2 test reports may result in unnecessary treatment of uninfected individuals that may further contribute to the consequences if affected individuals are involved in essential public services. Therefore, the present study was conducted with an aim to evaluate the errors and subsequent corrective actions during different phases of COVID-19 TrueNat testing in our health-care facility.

\section{MATERIALS AND METHODS}

The present study was carried out in the Department of Microbiology of a tertiary care center, Delhi over a period of 2 months, which is an ICMR approved center for COVID-19 testing by TrueNat system. The TrueNat testing system developed by MolBio Diagnostics Pvt., Ltd., Goa was used in the study. The samples for TrueNat testing were collected as per the ICMR, Ministry of Health and Family Welfare, Government of India guidelines for COVID-19 testing [12]. The oropharyngeal swab specimens in transport medium provided by the supplier were received in our specimen receiving unit of TrueNat testing lab for COVID-19. TrueNat system is based on the principle of rRT-PCR. TrueNat testing was performed as per the manufacturer's instructions. The envelope (E) gene of Sarbeco virus was targeted for screening test and if Beta-CoV was detected in the specimen, then a confirmatory test targeting the RNAdependent RNA polymerase (RdRp) gene was performed further from the extracted RNA using another TrueNat ${ }^{\mathrm{TM}}$ SARS CoV-2 chip. TrueNat system simultaneously detected the presence of human RNase $\mathrm{P}$ in each individual specimen in addition to target gene which served as an internal positive control (IPC) of the whole process of proper swab collection, RNA extraction, and amplification reaction.

The validity of each test run depends on the cycle threshold $(\mathrm{Ct})$ value of IPC. In negative specimens its absence or shift beyond pre-determined cut offs invalidated the individual test runs. In positive specimens with high viral load (low Ct values), even in the absence of IPC, test run was considered valid. The errors during different phases of TrueNat testing for COVID-19 were analyzed. The following information was collected: Number of samples tested by TrueNat system, number of samples with mismatched identification or specimen referral form (SRF) ID; number of insufficient samples received; number of samples received in unsuitable transport medium for TrueNat testing (transport medium other than the one supplied by TrueNat manufacturer); number of cartridge and chip errors encountered; number of failures in following the SOP during TrueNat testing for COVID19; number of tests with invalid runs; number of inconclusive tests; number of confirmed COVID-19 positive results; number of errors in reporting of results; and number of samples where reporting time exceeded beyond $24 \mathrm{~h}$ from the time of receiving of these samples at lab. The follow-up or corrective actions that were taken to address these testing errors were also recorded. The data recorded were analyzed using descriptive statistical tests.

\section{RESULTS}

Over a period of 2 months since the beginning of TrueNat testing for COVID-19 in our department, a total of 1108 samples were tested for COVID-19 by TrueNat system. Out of these, 82 (7.4\%) samples were confirmed positive (both E and RdRp genes detected) for SARS-CoV-2. COVID-19 test results were inconclusive (E gene detected, but RdRp not detected) in 13 $(1.2 \%)$ samples. A total of 112 testing errors were seen in the TrueNat testing of 1108 samples. Table 1 and Figure 1 show the distribution of errors during different phases of COVID-19 TrueNat testing. Majority of errors occurred in preanalytical phase of TrueNat testing, followed by analytical phase. 
Table 1: Distribution of errors during different phases of coronavirus disease 2019 TrueNat testing

\begin{tabular}{|c|c|c|}
\hline Phase of testing & Type of error & $\begin{array}{c}\text { Frequency of errors } \\
(\mathrm{n}=112)(\%)\end{array}$ \\
\hline \multirow[t]{5}{*}{ Preanalytical phase } & $\begin{array}{l}\text { Samples not collected } \\
\text { in TrueNat VTM }\end{array}$ & $8(7.14)$ \\
\hline & $\begin{array}{l}\text { Samples transported } \\
\text { without triple packaging }\end{array}$ & $1(0.89)$ \\
\hline & $\begin{array}{l}\text { Mismatched } \\
\text { identification/labeling }\end{array}$ & $5(4.46)$ \\
\hline & $\begin{array}{l}\text { Sample quantity } \\
\text { insufficient }\end{array}$ & $18(16.07)$ \\
\hline & $\begin{array}{l}\text { Internal positive control } \\
\text { failure }\end{array}$ & $34(30.36$ \\
\hline \multirow[t]{2}{*}{ Analytical phase } & $\begin{array}{l}\text { Failure of adherence } \\
\text { to standard operating } \\
\text { procedure }\end{array}$ & $2(1.79)$ \\
\hline & $\begin{array}{l}\text { Instrument } \\
\text { malfunctioning } \\
\text { (cartridge or chip error) }\end{array}$ & $42(37.50)$ \\
\hline $\begin{array}{l}\text { Post-analytical } \\
\text { phase }\end{array}$ & $\begin{array}{l}\text { Delay in reporting of } \\
\text { results }\end{array}$ & $2(1.79)$ \\
\hline
\end{tabular}

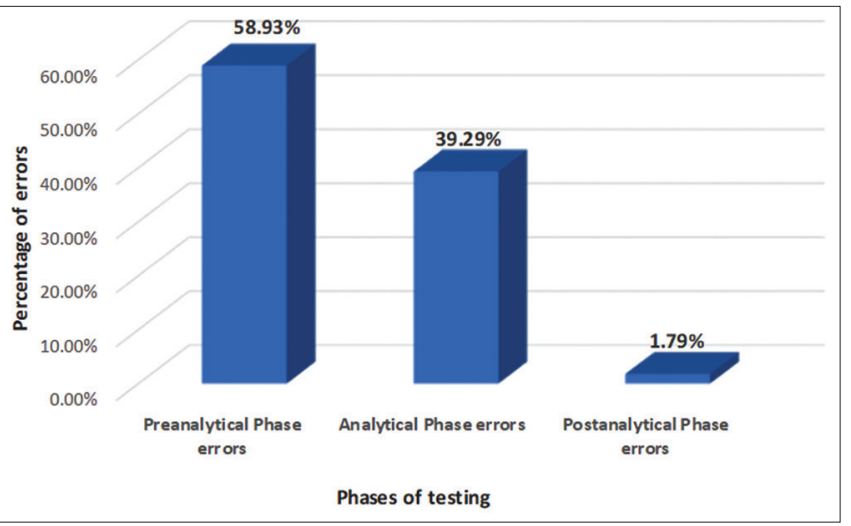

Figure 1: Distribution of errors during different phases of coronavirus disease 2019 TrueNat testing

During the preanalytical phase, $8(0.72 \%)$ samples were not collected in the TrueNat viral transport medium (VTM) and thus were unsuitable for TrueNat testing for COVID-19. As all these samples were collected in the VTM for conventional rRT-PCR testing, these were further directed to designated center for COVID-19 testing by conventional rRT-PCR testing as per institutional policy. One $(0.09 \%)$ sample was not transported with triple packaging. This sample was rejected as per the sample rejection criteria of our COVID-19 testing lab. The error of wrong labeling was observed in $5(0.45 \%)$ samples received in our lab for COVID-19 testing. The discrepancies were immediately conveyed to the concerned authorities and in four of these samples, the correct information was received. However, one sample had to be rejected in the lack of correct information.

The quantity of sample received was insufficient to permit TrueNat testing in $18(1.6 \%)$ samples. These samples with insufficient quantity were rejected as per the rejection criteria and repeat samples were requested from the same patients. Out of 18 samples rejected due to insufficient quantity, only 15 repeat samples from the same patients were received in our lab and in two of these repeat samples, SARS-CoV-2 was detected. In $34(3.1 \%)$ samples, there was a failure of IPC detection after a complete adherence to TrueNat system testing protocol. The TrueNat test runs in these cases were considered invalid and repeat samples were requested. On TrueNat testing of repeat samples, two samples were positive and 32 samples were negative for SARS CoV-2.

In the analytical phase, failure of adherence to SOP for COVID-19 testing by TrueNat system was observed in processing of $2(0.18 \%)$ samples. At one instance, error was encountered during dispensing of clear solution to TrueNat Beta CoV chip and at other instance, instead of loading a TrueNat SARS-CoV-2 chip for confirmatory testing after a positive screening test on TrueNat system, a repeat TrueNat Beta-CoV chip was loaded again. As failure had occurred after the viral RNA extraction step in both these cases, repeat testing was performed from the available extracted RNA. Instrument malfunctioning, cartridge or chip error, was seen in $42(3.79 \%)$ cases. Out of these 42 instances of instrument malfunctioning, 31 were cartridge errors and 11 were chip errors. All these samples were repeated with the fresh cartridge or chip as per the need.

In post-analytical phase, the entry of TrueNat testing report for COVID-19 on ICMR portal was delayed for $2(0.18 \%)$ samples. In both the cases, due to incomplete entries of patient details on ICMR portal during the generation of SRF number by the concerned clinical departments, final report of COVID19 TrueNat testing could not be submitted briskly. Concerned authorities were immediately informed and final COVID-19 TrueNat test report was submitted on the portal soon after the completion of details by the other end.

\section{DISCUSSION}

In the current scenario of ongoing pandemic of COVID-19, the diagnostic reliability of test reports is a foremost prerequisite. The current gold standard for SARS-CoV-2 detection is rRT-PCR on respiratory tract specimens [13-15]. Based on the principle of rRT-PCR, TrueNat testing has emerged as an important diagnostic modality for SARS-CoV-2. TrueNat testing can be performed with limited infrastructure and minimally trained manpower. COVID-19 test results by TrueNat testing technique become available in a short period of $1 \mathrm{~h}$. The dependability on TrueNat testing technique for the detection of COVID-19 cases at several COVID-19 testing labs emphasize on the need of better understanding of the testing errors that may occur in the various phases of COVID-19 TrueNat testing.

To ensure the diagnostic accuracy of any lab report, the emphasis is primarily laid on the analytical phase of testing. The preanalytical phase of testing that is crucial for a good quality specimen and subsequently for a reliable test result is very often underestimated $[16,17]$. In the present study, $58.93 \%$ of the total errors were observed during preanalytical 
phase of TrueNat testing for COVID-19. Our findings are in line with other studies that have also attributed the highest error rate to pre-analytic phase of testing [18-20]. However, none of these studies have evaluated any of the COVID-19 testing techniques. To the best of our knowledge, no other study has evaluated the errors during the various phases of COVID-19 lab testing.

In the present study, poor quality samples that were sent for COVID-19 TrueNat testing resulted in the failure of IPC during test run and this constituted the most common cause of error in preanalytical phase. The probable reason of this could be that the collection of oropharyngeal swab requires a close proximity to the patient and often leads to gag reflex. A faulty sample collection technique along with the associated fear of contracting COVID-19 in healthcare workers while collecting the samples might have resulted in poor quality samples. Another error observed during preanalytical phase of TrueNat testing for COVID-19 was use of inappropriate transport media for TrueNat testing. In these instances, VTM for conventional rRT-PCR was used instead of TrueNat transport medium. The simultaneous use of different testing techniques for the diagnosis of COVID-19 at one setup to handle the load of samples to be tested for COVID-19 in this pandemic situation might have led to this error.

Analytical phase errors contributed $39.29 \%$ of the total errors during COVID-19 TrueNat testing. The majority of these errors were the result of cartridge/chip failure. In this study, the delay in entry of COVID-19 TrueNat test results on ICMR portal contributed to errors in post-analytical phase of testing. The initial entry of patient details is done while generating the SRF number for each sample at the point of collection of samples for COVID-19 testing and the entry of final test report after TrueNat testing is performed by testing lab. As the information regarding patient details was incomplete, the test report could not be submitted on portal at laboratory end immediately after obtaining the result. While considering the common perception that at least analytical phase of testing remains under the strict control of testing lab and errors during this phase can be reduced by standardization of testing techniques and instruments, with adherence to quality control and quality assurance methods [21].

In the present study, the unanticipated errors in cartridge and chip were beyond the control of TrueNat testing lab. Although errors in analytical phase of TrueNat testing had not affected the final report, these have caused a comparative delay in release of individual test results in addition to already high workloads in the face of COVID-19 emergency. Even the post-analytic phase of COVID-19 testing involves the synchronous efforts at various levels. The present research had few limitations including, short duration study with limited number of samples at a single center of COVID-19 testing by TrueNat system. We recommend more exhaustive studies involving multiple testing centers with larger sample size in future to further validate our research.

\section{CONCLUSION}

The current pandemic of COVID-19 has immensely magnified the vulnerability of laboratory medicine. With limited manpower and logistics, HCWs including laboratory staff are facing towering workloads under severe pressure. As the susceptibility to testing errors enhance multifold in such trying conditions, so does the repercussions to these errors. The accuracy of COVID-19 test reports is extraordinarily important as any fallacy not only endangers the health of an individual but may also undermine the efficacy of local, national, and international measures taken for the control of COVID-19 pandemic. The only way to reduce errors in various phases of COVID-19 testing is to analyze them thoroughly for their better understanding and subsequently follow them up with effective corrective actions.

\section{REFERENCES}

1. Ministry of Health and Welfare, Government of India. COVID-19 India. Available from: https://www.mohfw.gov.in. [Last accessed on 2020 Sep 17].

2. World Health Organization. FIFA/COVID-19 Virtual Press Conference; 2020. Available from: https://www.who.int/docs/default-source/ coronaviruse/transcripts/who-audio-emergencies-coronavirus-pressconference-full-23mar2020.pdf?sfvrsn=846ecb41_4. [Last accessed 2020 Sep 02].

3. Indian Council of Medical Research Department of Health Research, Ministry of Health and Family Welfare, Government of India. Total Operational (Initiated Independent Testing) Laboratories Reporting to ICMR. Available from: https://www.icmr.gov.in/pdf/covid/labs/COVID_ Testing_Labs_16092020.pdf. [Last accessed on 2020 Sep 17].

4. Indian Council of Medical Research Department of Health Research, Ministry of Health and Family Welfare, Government of India. SARS-CoV-2 (COVID-19) Testing Status. Available from: https://www.icmr.gov.in/pdf/ covid/update/ICMR_testing_update_17Sept2020.pdf. [Last accessed on 2020 Sep 17].

5. Indian Council of Medical Research Department of Health Research, Ministry of Health and Family Welfare, Government of India. Guidance on the Use of TruenatTM Beta CoV. Available from: https://www.icmr.nic.in/ sites/default/files/upload documents/Guidance TrueNat 14042020.pdf. [Last accessed on 2020 Sep 04].

6. Ravichandran K, Anbazhagan S, Singh SV, et al. Global status of COVID-19 diagnosis: An overview. J Pure Appl Microbiol 2020;14:879-2.

7. Indian Council of Medical Research Department of Health Research, Ministry of Health and Family Welfare, Government of India. Revised Guidelines for TrueNat Testing for COVID-19. Available from: https://www.icmr.gov.in/ pdf/covid/labs/Revised_Guidelines_TrueNat_Testing_19052020.pdf. [Last accessed on 2020 Sep 09].

8. Lippi G, Simundic AM, Plebani M. Potential preanalytical and analytical vulnerabilities in the laboratory diagnosis of coronavirus disease 2019 (COVID-19). Clin Chem Lab Med 2020;58:1070-6.

9. Lippi G, Plebani M. A Six-Sigma approach for comparing diagnostic errors in healthcare-where does laboratory medicine stand? Ann Transl Med $2018 ; 6: 180$.

10. Sheridan C. Coronavirus and the race to distribute reliable diagnostics. Nat Biotechnol 2020;38:382-4.

11. Lippi G, Plebani M, Graber ML. Building a bridge to safe diagnosis in health care. The role of the clinical laboratory. Clin Chem Lab Med 2016;54:1-3.

12. ICMR Guidelines for COVID-19 Testing. Advisory on Strategy for COVID-19 Testing in India. Available from: https://www.icmr.gov.in/pdf/ covid/strategy/Testing_Strategy_v6_04092020.pdf. [Last accessed on 2020 Sep 04].

13. Lippi G, Plebani M. The novel coronavirus (2019-nCoV) outbreak: Think the unthinkable and be prepared to face the challenge. Diagnosis (Berl) 2020;7:79-81.

14. Pang J, Wang MX, Ang IY, et al. Potential rapid diagnostics, vaccine and therapeutics for 2019 novel coronavirus (2019-nCoV): A systematic review. 
J Clin Med 2020;9:623.

15. Jin YH, Cai L, Cheng ZS, et al. A rapid advice guideline for the diagnosis and treatment of 2019 novel coronavirus (2019-nCoV) infected pneumonia (standard version). Mil Med Res 2020;7:4.

16. Lippi G, Von Meyer A, Cadamuro J, et al. PREDICT: A checklist for preventing preanalytical diagnostic errors in clinical trials. Clin Chem Lab Med 2020;58:518-26.

17. Lippi G, Salvagno GL, Mattiuzzi C. Public perception of diagnostic and laboratory errors among Internet users. Diagnosis (Berl) 2019;6:385-6.

18. Plebani M. Exploring the iceberg of errors in laboratory medicine. Clin Chim Acta 2009;404:16-23.

19. Howanitz PJ. Errors in laboratory medicine: Practical lessons to improve patient safety. Arch Pathol Lab Med 2005;129:1252-61.

20. Carraro P, Zago T, Plebani M. Exploring the initial steps of the testing process: Frequency and nature of pre-preanalytic errors. Clin Chem 2012;58:638-42.

21. Plebani M. Diagnostic errors and laboratory medicine-causes and strategies. EJIFCC 2015;26:7-14.

Funding: None; Conflicts of Interest: None Stated.

How to cite this article: Goyal N, Sharma A, Loomba P, Mishra B, Thakur A. Evaluation of errors during different phases of coronavirus disease 2019 TrueNat testing in a tertiary care hospital. East J Med Sci. 2020;5(4):79-83.

Doi: 10.32677/EJMS.2020.v05.i04.003 